\title{
Ultrafast Direct Peeling Method for Fabrication of High Aspect Ratio Superhydrophobic Nanohair Structure
}

\author{
Pei-Shiang Chen, Han-Hsun Chang, Chiu-Chang Huang, Jun-Wei Chen, \\ Hsiang-Lin Liu", Yu-Hsin Lin², Yi-Chiuen Hu², Kai-Yuan Chi, and Chih-Yu Chao* \\ Department of Physics, National Taiwan University, Taipei 10617, Taiwan \\ ${ }^{1}$ Department of Physics, National Taiwan Normal University, Taipei 11677, Taiwan \\ ${ }^{2}$ Instrument Technology Research Center, National Applied Research Laboratories, Hsinchu 30076, Taiwan \\ Received October 2, 2008; accepted December 17, 2008; published online March 23, 2009
}

In this paper, we present an ultra-fast, simple and cost-effective way - the direct peeling method (DPM) - to produce nanohairs with high aspect ratio on fluorinated ethylene propylene (FEP) films within $30 \mathrm{~s}$. The FEP films with nanohair structures afford impressive demonstrations of superhydrophobicity and self-cleaning effect; the characteristics described above along with the flexibility of FEP may prove useful for solar cells and curved components in the field of biological and technological applications.

(C) 2009 The Japan Society of Applied Physics

\section{Introduction}

Nanohair structures have been found to play an important role among living creatures. The subwavelength antireflective feature on cicada wings is caused by hair-like structures. ${ }^{1)}$ Geckos rely on nanohairs to adhere to ceilings and vertical walls. ${ }^{2)}$ The superhydrophobic property and self-cleaning behavior on lotus leaves stem from hierarchical nanohair structures along with a hydrophobic wax coating ${ }^{3-5)}$ since that contaminating particles such as water droplets and dirt can only lie on the tips of nanohair structures, they can barely develop strong adhesion forces and can be removed effectively by rain. Because of the decent characteristics stated above, the biological nanohair structures and functions have been applied to the design of man-made mimics technologically.

During the past few years, multiple attempts have been reported to manufacture nanohairs. Here, we take some of them for example. (i) The most traditional one is to use photolithography or electron beam (E-beam) lithography followed by etching process to make nanohair structures. ${ }^{6-8)}$ (ii) Via applying electric field created by biased voltage between oxygen plasma and substrate to form a gradient, nanohairs will be drawn from polymer surfaces naturally because of dielectric property. ${ }^{9)}$ (iii) Nanohairs can be fabricated by capillarity-driven molding combined with elongation at mold interface. ${ }^{10)}$ (iv) By means of photolithography or E-beam lithography followed by chemical vapor deposition (CVD), carbon nanotubes will grow spontaneously on a silicon substrate. ${ }^{1-15)}$ (v) Pouring poly(methyl methacrylate) (PMMA), poly(dimethylsiloxane) (PDMS), or polystyrene (PS) to molds made up of wax, photoresist or alumina template to create nanohairs. ${ }^{16-19)}$ (vi) Melting polymers or filling low viscosity precursors to nanoporous membranes such as anodic aluminum oxide (AAO) and polycarbonate filter and then dissolving them in solutions, such as hydrochloric acid $(\mathrm{HCl})$ and methylene chloride $\left(\mathrm{CH}_{2} \mathrm{Cl}_{2}\right){ }^{20,21)}$

Although nanohairs can be generated through all the approaches described above, there are certain kinds of limitations of these methods. First, we can hardly generate

*E-mail address: cychao@phys.ntu.edu.tw hairs with large enough aspect ratio (AR) via (i) and (v). Second, (ii) is so hard to control that nanohairs cannot be aligned efficiently. Third, (iv) requires manufacturing processes which are too complicated to follow. Finally, although (iii) and (vi) can both be feasible ways to create hairs with large AR, (iii) needs to be conducted under continuing high temperature for more than one hour and the membranes in (vi) can only be used once.

Here, we present a simple and novel way by which nanohairs with high AR and good alignment can be created within $30 \mathrm{~s}$. Via applying instant high pressure and instant heating temperature to the polymer layer, the adhesive force forming between the polymer and mold will enable nanohairs with large AR to generate as soon as we peel the polymer layer off the mold quickly. Here, we go through only two steps - applying temperature as well as pressure and peeling off the polymer - in $30 \mathrm{~s}$, which proves to be much more efficient and less complicated than any other existing methods. Moreover, from scanning electron microscope (SEM) images, the nanohair structure was found to possess great alignment over a macroscopic area of at least $2.6 \mathrm{~cm}^{2}$. The molds can be used for more than 20 times in this approach, which is more cost-effective than most of the other methods.

In this study, we chose fluorinated ethylene propylene (FEP) to be the polymer base, which is another focus in addition to direct peeling method (DPM). FEP is a copolymer of tetrafluoroethylene (TFE) using hexafluoropropylene (HFP); the glass transition temperature $\left(T_{\mathrm{g}}\right)$ is $260-280^{\circ} \mathrm{C}$ and the elastic modulus is 70000 pound per square inch (PSI) at room temperature; its elongation can reach $300 \%$. In addition to decent properties such as high/low temperature resistance and acid/base proof, its hydrophobic surface may transfer to a superhydrophobic structure right after suitable nanohair structures are created on it; thus, the superhydrophobicity resulted from the characteristics of nanohair structures $^{22,23)}$ will prove useful as a self-cleaning layer on the surface of solar cells, windshields, windows of buildings or other devices.

\section{Experiments}

Figure 1 outlines the schematic illustration of DPM. In order to form the negative mold necessary in the process, we used 


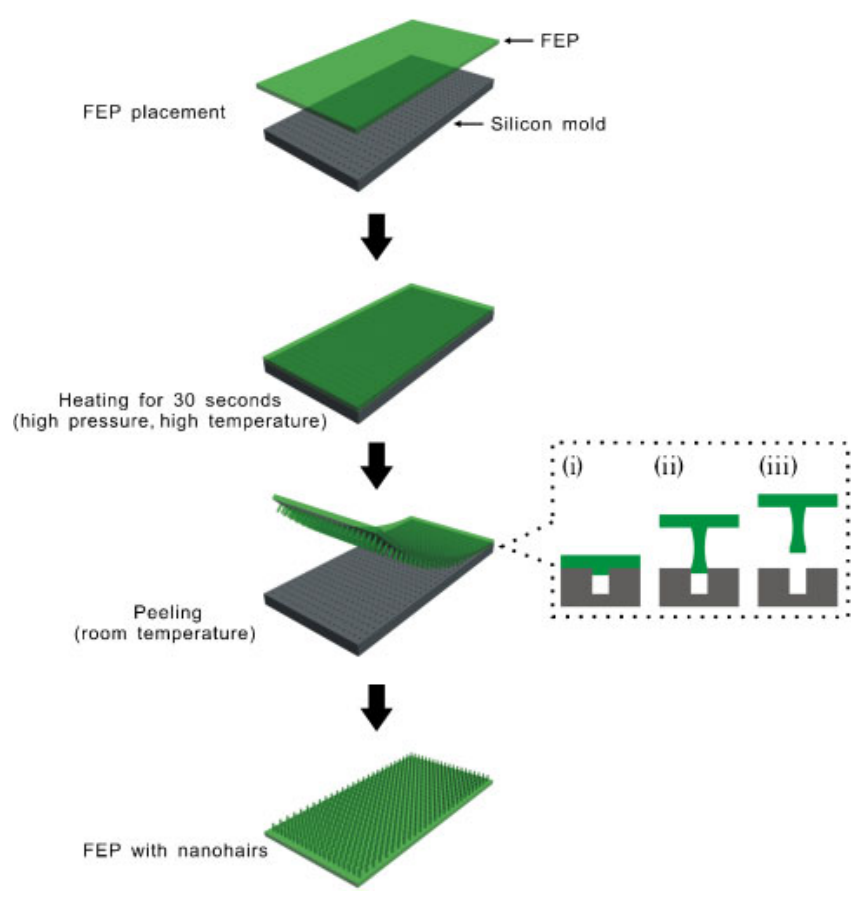

Fig. 1. (Color online) The schematic illustration of the DPM. First, we apply high temperature and pressure to the sample for about $30 \mathrm{~s}$. Afterwards, we manually peel off the FEP film at room temperature, resulting in nanohairs with high AR.

deep ultraviolet (UV) lithography to pattern a hole-array on a silicon wafer; later on, through inductively coupled plasma reactive ion etching (ICP-RIE), the wafer was etched and holes with depth were created. In our research, we have used two molds: mold 1 is $5.0 \mu \mathrm{m}$ in depth and $840 \mathrm{~nm}$ in width with a $2 \mu \mathrm{m}$ pitch; mold 2 is $3.3 \mu \mathrm{m}$ in depth and $640 \mathrm{~nm}$ in width with a $1 \mu \mathrm{m}$ pitch. After the silicon mold was prepared, the FEP film ( $\sim 125 \mu \mathrm{m}$ thickness, Dupont) was placed on the mold and the sample as a whole was put in a vacuum chamber. Via nitrogen gas ( $\sim 99 \%$ concentration) with high pressure and tungsten lamp used for heating, we applied high temperature (larger than $170^{\circ} \mathrm{C}$ ) and pressure (>120 PSI) to the sample for about $30 \mathrm{~s}$. Afterwards, we manually peeled off the FEP film at a very high velocity at room temperature, resulting in nanohairs with high AR (>17) and good alignment over a large area. Figure 1(i)-(iii) is the schematic illustration of the changes in nanohair structures during the peeling procedure. Because of capillary force, FEP filled into the mold's cavities slightly when we applied instant heat and pressure. When FEP was cooled down to room temperature, the FEP already filled into the mold would cause an adhesive force on the surface of hole cavities. During the peeling process, the mold-untouched FEP was elongated to create the long-straight part of nanohairs and the mold-touching FEP resulted in the mushroom structures on the tip of nanohairs; as long as our peeling force was larger than the adhesive force, the mold-touching FEP would be separated from the mold; the high AR nanohair structures with mushroom structures on the tip would be created right afterwards. In this experiment, we manually peeled several samples under the same experimental conditions but with different speeds and we found that the variations of nanohair in height and width were not obvious. This result indicates that the configuration of nanohairs does not depend or only slightly depend on peeling speed of a FEP film.

\section{Results and Discussion}

Figure 2 shows the SEM images of FEP nanohairs created through DPM. Figure 2(a) is a large area of elongated nanohair structures fabricated from mold 1 (depth: $5.0 \mu \mathrm{m}$; width: $840 \mathrm{~nm}$ ). Figure 2(b) is the magnified view of Fig. 2(a). Likewise, Fig. 2(c) is a large area of elongated nanohair structures fabricated from mold 2 (depth: $3.3 \mu \mathrm{m}$; width: $640 \mathrm{~nm}$ ) and Fig. 2(d) is the magnified view of Fig. 2(c). Moreover, in Figs. 2(b) and 2(d), it is obvious that there is a mushroom structure on the tip of nanohairs. The mushroom structure can be viewed as a proof that FEP only filled into the hole cavity slightly and adhesive force could only form and function in a very small area.

Figure 3 presents the changes in hair width and length under a series of temperature, pressure and heating time conditions. Figure 3(a) presents the relation between heating time and hair length as well as hair width in samples fabricated from mold 2 under heating temperature $210^{\circ} \mathrm{C}$ and 300 PSI pressure. As shown in the diagram, the length of nanohairs ranged from 5.5 to $13.0 \mu \mathrm{m}$. Since that the FEP filling into the hole cavity because of capillary force increases with time, the adhesive force strengthens simultaneously; thus, longer nanohairs are produced by elongation as we peel off the FEP film. However, the width of the longstraight part of nanohairs did not change obviously with time. It remained at about $325 \mathrm{~nm}$, which is much thinner than the width of holes. Figure 3(b) shows that the average aspect ratio increased from 17.0 to 38.4 as heating time went by; thus, in DPM, we can produce nanohairs with different high aspect ratios by controlling heating time.

In addition, when heating time was as short as $30 \mathrm{~s}$, which is much shorter than that needed for FEP to fill in the mold completely, it is supposed that only a small amount of FEP could fill in the mold; however, the length of resulting nanohairs was longer than the depth of the mold. Therefore, the depth of the mold is not one of the most important factors to determine the length of nanohairs while the adhesive force forming on the contacting surface of the hole and FEP plays a rather important role. In short, via DPM, we can create nanohairs with high AR without molds with deep holes in a very short period of time.

Figure 3(c) demonstrates the relation between pressure and hair length as well as hair width in samples fabricated from mold 2 under $210^{\circ} \mathrm{C}$ and heating time $30 \mathrm{~s}$. It is known from the diagram that the length and width of nanohairs do not vary obviously with pressure and that the influence of applied pressure is nearly negligible. Since that the adhesive force is the main determinant of nanohair length and the instant high pressure seems to have very slight influence on it, the applied pressure cannot cause the length to alter obviously. As for the hair width, it is similar to that presented in Fig. 3(a), an average of about $325 \mathrm{~nm}$.

Figure 3(d) presents the relation between heating temperature and hair length as well as hair width in samples fabricated from mold 1 under $30 \mathrm{~s}$ and 300 PSI pressure. As is shown in the graph, there is no significant change in the 


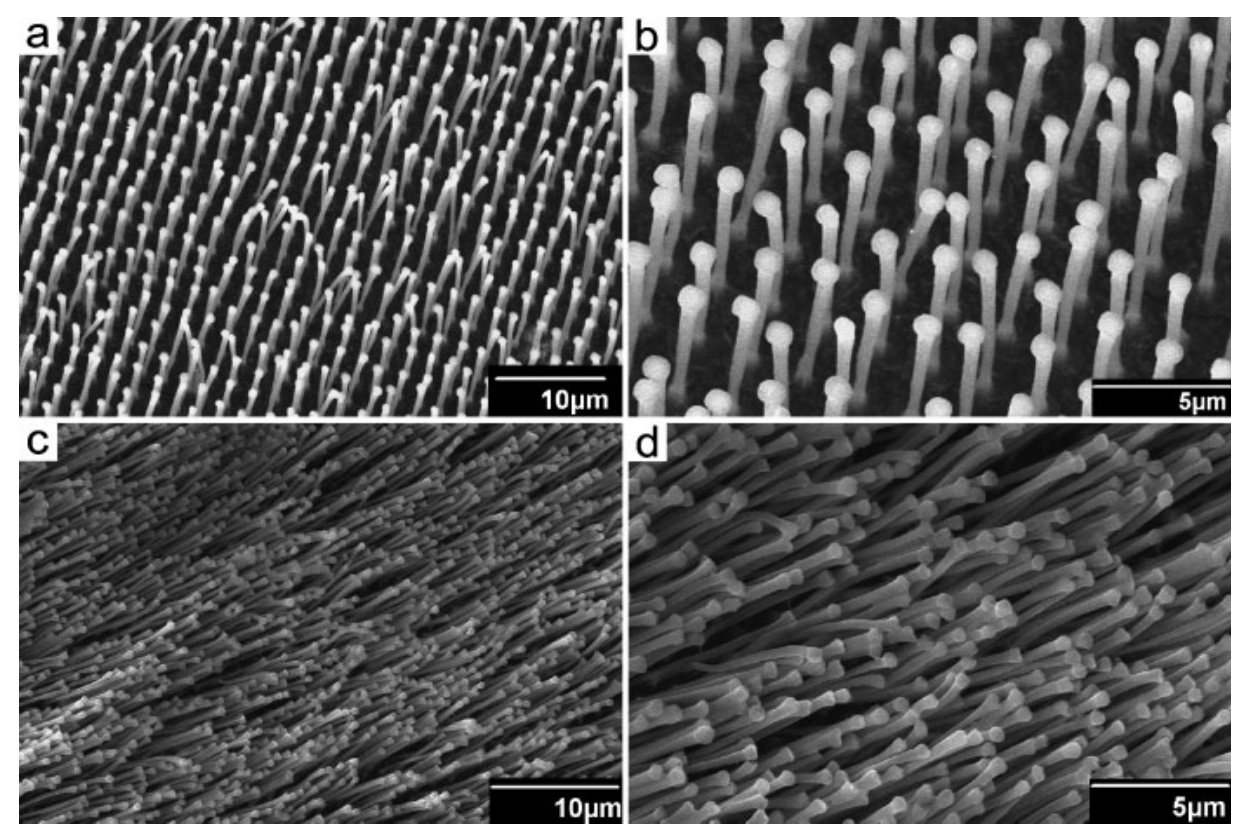

Fig. 2. (a) An SEM image of a large area of elongated nanohairs fabricated from mold 1. (b) The magnified view of (a). (c) An SEM image of a large area of elongated nanohairs fabricated from mold 2. (d) The magnified view of (c).

a

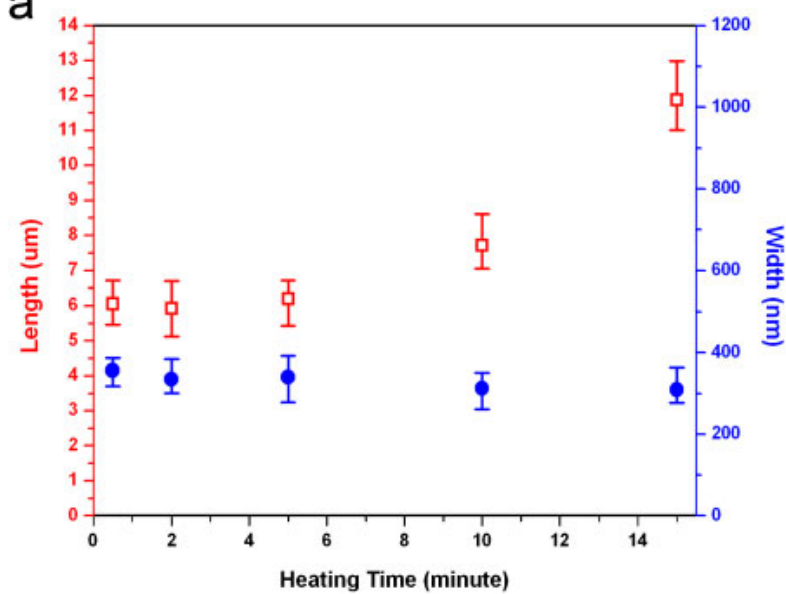

C

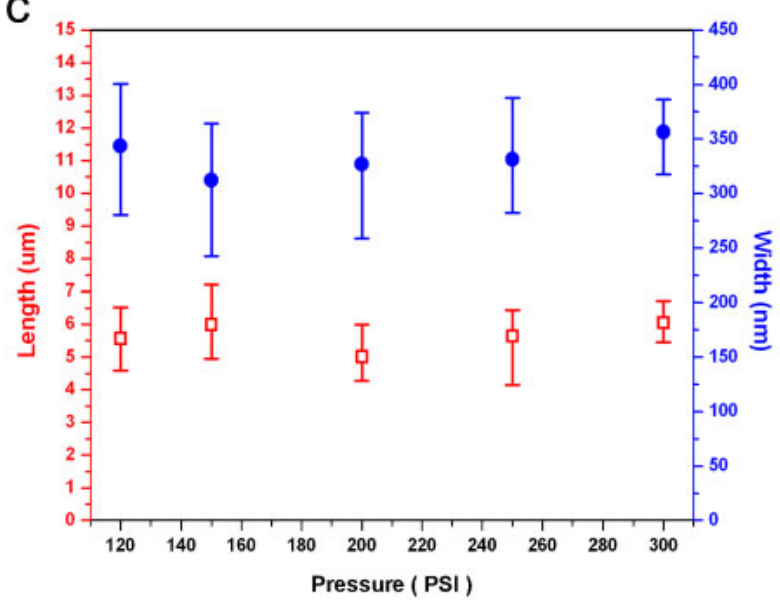

b

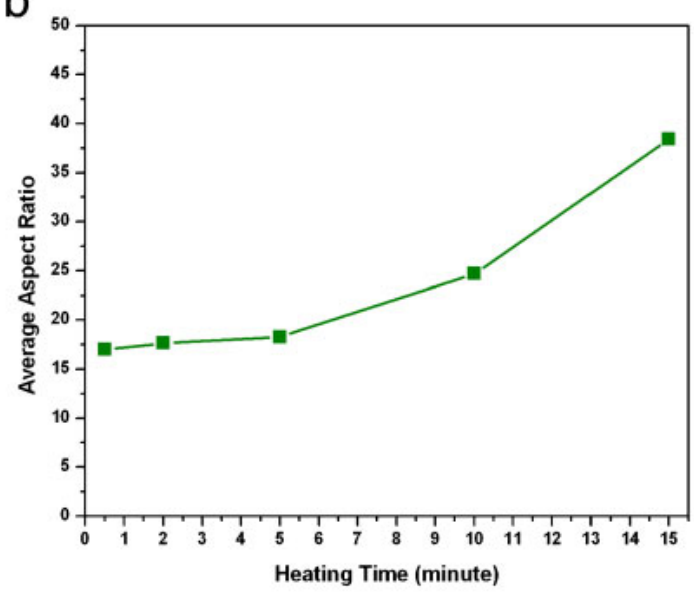

d

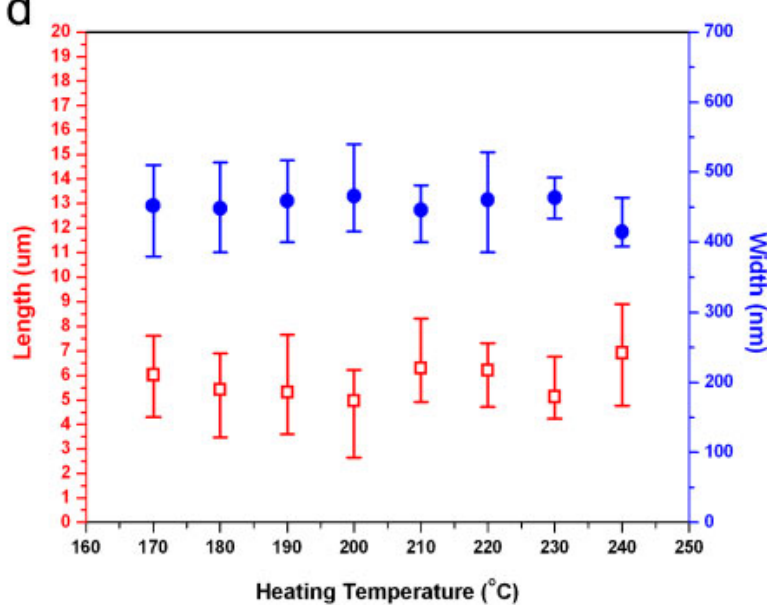

Fig. 3. (Color online) (a) The diagram of the relation between heating time and hair length as well as hair width in samples fabricated from mold 2 (depth: $3.3 \mu \mathrm{m}$; width: $640 \mathrm{~nm}$ ) under $210^{\circ} \mathrm{C}$ and $300 \mathrm{PSI}$. (b) The diagram of the relation between average aspect ratio and heating time in samples fabricated from mold 2 under $210^{\circ} \mathrm{C}$ and $300 \mathrm{PSI}$. (c) The diagram of the relation between pressure and hair length as well as hair width in samples fabricated from mold 2 under $210^{\circ} \mathrm{C}$ and heating time $30 \mathrm{~s}$. (d) The diagram of the relation between heating temperature and hair length as well as hair width in samples fabricated from mold 1 (depth: $5.0 \mu \mathrm{m}$; width: $840 \mathrm{~nm}$ ) under $300 \mathrm{PSI}$ and heating time $30 \mathrm{~s}$. 
length and width of nanohairs. In other words, the instant high temperature can barely influence the length and width of nanohairs. However, the average width of nanohairs was $450 \mathrm{~nm}$, which is different from that shown in Figs. 3(a) and 3 (c), where the measured sample was fabricated from mold 2 with $640 \mathrm{~nm}$ in width.

When we compared the Figs. 3(c) and 3(d), we can see that the nanohair length in Figs. 3(c) and 3(d) is almost the same while the samples were fabricated from different molds with distinct depths. In other words, different kinds of mold can result in the same nanohair length. Therefore, we have proved from the results again that the depth of the mold is not one of the important factors to determine the length of nanohairs.

In Fig. 3(a) to 3(d), we have demonstrated three factors - heating time, temperature and pressure - which might influence the resulting length and width of nanohairs. From the results, we discovered that only heating time can cause the changes in the length of nanohairs while heating temperature and pressure could not. This is because nanohair length is highly in correlation with adhesive force, and the heating time rather than the instant heating temperature or pressure is the most important determinant of adhesive force. As for the width of nanohairs, it is reasonable to derive that the main determinant is the width of hole cavity. In Fig. 3(c), the nanohair width is $325 \mathrm{~nm}$, which is about half of the width of the hole $(640 \mathrm{~nm})$; in Fig. 3(d), the nanohair width is $450 \mathrm{~nm}$, about half of the width of the hole $(840 \mathrm{~nm})$; in Figs. 3(c) and 3(d), it is presented that instant heating temperature and pressure have no significant influence on the width of nanohairs. Therefore, the width of the hole cavity is obviously the main factor that influence the width of nanohairs.

Figure 4 presents the relation between contact angle and nanohair head width. The contact angle was measured via a contact angle meter (KRÜSS GH-100). The volume of the distilled water droplet placed on the nanohairs was $10 \mu \mathrm{L}$. In Fig. 4(a), the deionized (DI) water is dropped on the flat FEP; the contact angle is found to be about $108.0^{\circ}$, which proves that FEP is highly hydrophobic itself. Therefore, we do not need subsequent hydrophobizing treatment such as coating hydrophobic layers in order to achieve large contact angle. Figure 4(b) is the picture of DI water dropped on FEP with nanohair structures fabricated from mold 2 ; as shown in this photograph, the measured contact angle is obviously larger than $136.0^{\circ}$. The maximum static apparent contact angle can reach $151.8^{\circ}$ in our experiments, which indicates the superhydrophobic property of the combination of nanohair structures and FEP films.

In this case, for the high AR nanohairs made via DPM, air pockets were trapped in the spaces between nanohairs, which resisted the depression of a droplet into the structure. Thus, the apparent contact angle $\theta_{\mathrm{D}}$ is given by the CassieBaxter equation for wetting on composite surfaces made of the solid and air: ${ }^{24)}$

$$
\cos \theta_{\mathrm{D}}=f_{1} \cos \theta+f_{1}-1,
$$

where $f_{1}$ is the ratio of the actual area of liquid-solid contact interface to the projected area on the horizontal plane of the rough surface and $\theta$ is the equilibrium contact angle of the
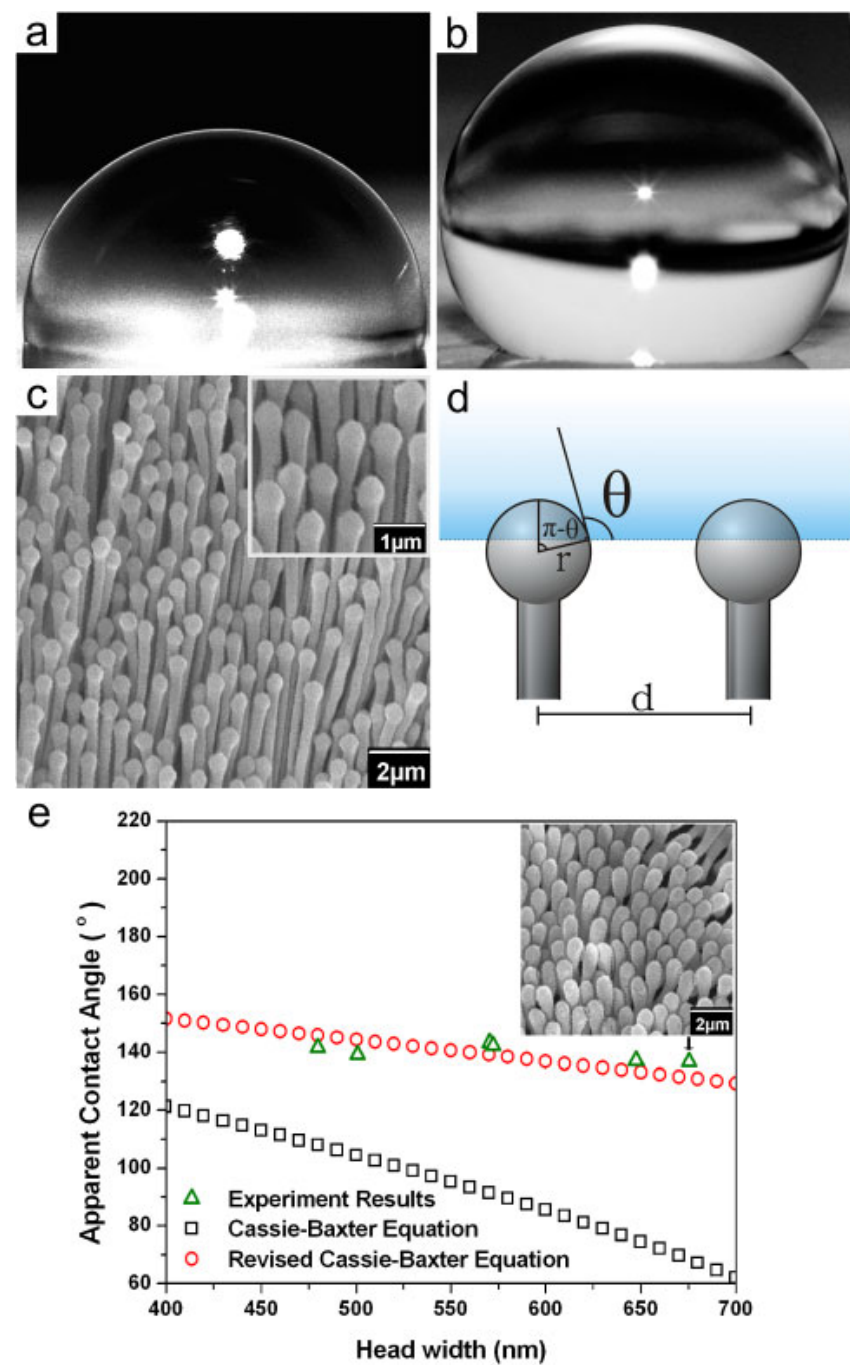

Fig. 4. (Color online) (a) The digital camera image of a DI water dropped on the flat FEP. The contact angle here is about $108.0^{\circ}$, proving that FEP is highly hydrophobic itself. (b) The digital camera image of DI water dropped on FEP with nanohair structures fabricated from mold 2. The maximum static apparent contact angle can reach $151.8^{\circ}$. (c) The SEM image of nanohairs on the FEP film. (d) A schematic illustration of the spherical ball assumption for the nanohair tip in (c). (e) The diagram of the relation between apparent contact angle and head width of the nanohairs based on Cassie-Baxter equation (open squares) and revised Cassie-Baxter equation (open circles). Our experimental data are shown as open triangle symbols. Inset is an SEM image of nanohair structures on the FEP film under $250^{\circ} \mathrm{C}$ temperature for $1 \mathrm{~h}$.

liquid drop on the corresponding flat surface. According to our observations [see Fig. 4(c)], first we assume that there is a sphere of $r \mathrm{~nm}$ in diameter [see Fig. 4(d)] on the tip of nanohairs; the head width of the nanohair tip is $r \mathrm{~nm}$, the pitch of the nanohairs $d$ is $1 \mu \mathrm{m}$, and the $\theta$ of FEP is about 108. $0^{\circ}$ [known from Fig. 4(a)]. In Fig. 4(d), based on the spherical ball assumption, the liquid-solid contact interface is a semi-sphere with an angle $\pi-\theta$, and the projected area on the horizontal plane of the rough surface is $d^{2}$. We can derive that

$$
f_{1}=\frac{\int_{0}^{2 \pi} \int_{0}^{\pi-\theta} r d \theta r \sin \theta d \phi}{d^{2}}=\frac{2 \pi r^{2}(1+\cos \theta)}{d^{2}} .
$$


Via inserting $f_{1}$ into eq. (1), we can get the relation between theoretical apparent contact angle and the head width of the nanohairs. In Fig. 4(e), the square spot indicates the trend of the Cassie-Baxter equation; the triangle spot demonstrates our experimental results, most of which are larger than the theoretical value. The circle spot presents the revised Cassie-Baxter equation where $r$ is replaced by $0.5 r$ in the Cassie-Baxter equation. We conducted this revise because the tip of the nanohair is not exactly a right spherical ball, but an elliptic volume, where the actual $r$ is smaller than the width of the head and the actual liquid-solid contact area (the numerator in $f_{1}$ ) is smaller than that we assume. According to the revised Cassie-Baxter equation, the contact angles are larger than those in the original one, which fit our experimental results better in a reasonable way; the contact angle increases as the head width decreases, which indicates that the smaller the head width, the larger the contact angle. In other words, we can use molds with a smaller width of hole cavities to fabricate nanohairs with small head width, resulting in large contact angles.

In order to understand the phenomenon of nanohairs under high temperature, we put the FEP with nanohair structures in the oven at $250^{\circ} \mathrm{C}$ for $1 \mathrm{~h}$. From the inset of Fig. 4(e), we found that even under $250^{\circ} \mathrm{C}$ for $1 \mathrm{~h}$, the hair array did not melt or collapse; however, the width of nanohairs as a whole became a little larger [see inset of Fig. 4(e)] than that before, but the contact angle still remained larger than $136.0^{\circ}$. This proves that large contact angles can be maintained even on heated FEP with nanohair structures and FEP with nanohair structures truly possesses good heat-resist ability.

To demonstrate the self-cleaning ability of the FEP film with nanohairs, we soiled these films with silica particles $3 \mu \mathrm{m}$ in diameter [see Fig. 5(a)]. The silica particles here represent dust. When FEP films were rinsed with deionized (DI) water, the water droplets rolled off easily and carried most of the silica particles. Figure 5(b) is the cleaned FEP surface after rinsed with water. It is shown from the photograph that the synthetic FEP film with nanohair structures possess great self-cleaning ability.

\section{Conclusions}

In conclusion, we devised an ultra-fast and effective waythe direct peeling method (DPM) - to produce nanohairs with high AR on FEP films. The main determinants of nanohair length and width are heating time and the width of hole cavities. Here, the combination of FEP and the nanohair structures fabricated via DPM possesses superhydrophobicity without any subsequent hydrophobizing treatment on account of the hydrophobic property of FEP. And the large contact angle of DI water droplets leads to the self-cleaning characteristic. Because of the good flexibility, great solar transmission, extreme-temperature proof and outstanding chemical resistance, the FEP film with nanohair structures may be suitable for solar cells, windshields, windows of buildings, and other curved components in the field of biological and technological applications.

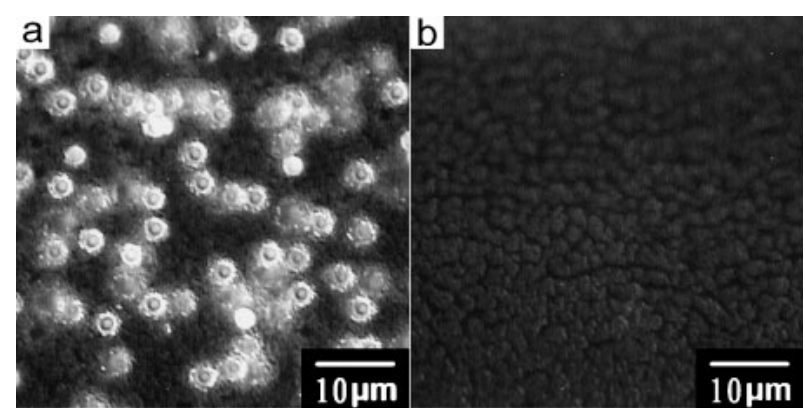

Fig. 5. (a) An optical microscope photograph of the FEP film soiled with silica particles $3 \mu \mathrm{m}$ in diameter. (b) An optical microscope photograph of the FEP film after rinsed with DI water.

\section{Acknowledgments}

One of us (C.Y.C.) acknowledges the support from the National Science Council, the Ministry of Education of Taiwan, and the National Nano Device Laboratories of Taiwan.

1) G. Xie, G. Zhang, F. Lin, J. Zhang, Z. Liu, and S. Mu: Nanotechnology 19 (2008) 095605.

2) A. M. Peattie and R. J. Full: Proc. Natl. Acad. Sci. U.S.A. 104 (2007) 18595.

3) W. Barthlott and C. Neinhuis: Planta 202 (1997) 1.

4) Y. T. Cheng, D. E. Rodak, A. Angelopoulos, and T. Cacek: Appl. Phys. Lett. 87 (2005) 194112

5) Y. T. Cheng, D. E. Rodak, C. A. Wong, and C. A. Hayden: Nanotechnology 17 (2006) 1359

6) A. K. Geim, S. V. Dubonos, I. V. Grigorieva, K. S. Novoselov, A. A. Zhukov, and S. Y. Shapoval: Nat. Mater. 2 (2003) 461.

7) R. Fürstner, W. Barthlott, C. Neinhuis, and P. Walzel: Langmuir 21 (2005) 956

8) M. T. Northen and K. L. Turner: Sens. Actuators A 130-131 (2006) 583.

9) M. T. Northen and K. L. Turner: Curr. Appl. Phys. 6 (2006) 379.

10) H. E. Jeong, S. H. Lee, P. Kim, and K. Y. Suh: Nano Lett. 6 (2006) 1508.

11) Y. Zhao, T. Tong, L. Delzeit, A. Kashani, M. Meyyappan, and A. Majumdar: J. Vac. Sci. Technol. B 24 (2006) 331.

12) K. Gjerde, J. Kjelstrup-Hansen, C. H. Clausen, K. B. K. Teo, W. I. Milne, H. G. Rubahn, and P. Bøggild: Nanotechnology 17 (2006) 4917.

13) L. Qu and L. Dai: Adv. Mater. 19 (2007) 3844.

14) L. Ge, S. Sethi, L. Ci, P. M. Ajayan, and A. Dhinowala: Proc. Natl. Acad. Sci. U.S.A. 104 (2007) 10792.

15) B. Aksak, M. Sitti, A. Cassell, J. Li, M. Meyyappan, and P. Callen: Appl. Phys. Lett. 91 (2007) 061906.

16) E. S. Yoon, R. A. Singh, H. Kong, B. Kim, D. H. Kim, H. E. Jeong, and K. Y. Suh: Tribology Lett. 21 (2006) 31.

17) S. Reddy, E. Arzt, and A. del Campo: Adv. Mater. 19 (2007) 3833.

18) B. Aksak, M. P. Murphy, and M. Sitti: Langmuir 23 (2007) 3322.

19) C. Greiner, A. del Campo, and E. Arzt: Langmuir 23 (2007) 3495.

20) C. Majidi, R. E. Groff, Y. Maeno, B. Schubert, S. Baek, B. Bush, R. Maboudian, N. Gravish, M. Wilkinson, K. Autumn, and R. S. Fearing: Phys. Rev. Lett. 97 (2006) 076103.

21) T. S. Kustandi, V. D. Samper, W. S. Ng, A. S. Chong, and H. Gao: J. Micromech. Microeng. 17 (2007) N75.

22) S. Sethi, L. Ge, L. Ci, P. M. Ajayan, and A. Dhinojwala: Nano Lett. 8 (2008) 822.

23) Y. J. Lee, D. S. Ruby, D. W. Peters, B. B. McKenzie, and J. W. P. Hsu: Nano Lett. 8 (2008) 1501.

24) A. B. D. Cassie and S. Baxter: Trans. Faraday Soc. 40 (1944) 546. 